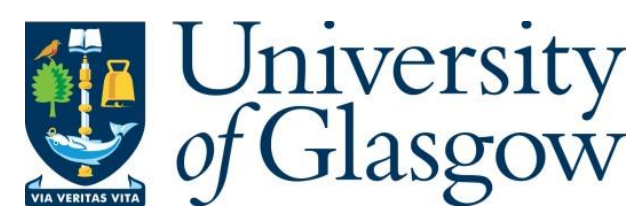

Li, J., Heidari, H., Shi, J. and Wang, Y. (2019) Magnetic Crosstalk Suppression and Probe Miniaturization of Coupled Core Fluxgate Sensors. In: IEEE Sensors 2019, Montreal, QC, Canada, 27-30 Oct 2019, ISBN 9781728116341.

There may be differences between this version and the published version. You are advised to consult the publisher's version if you wish to cite from it.

http://eprints.gla.ac.uk/192777/

Deposited on: 12 August 2019

Enlighten - Research publications by members of the University of Glasgow http://eprints.gla.ac.uk 


\title{
Magnetic Crosstalk Suppression and Probe Miniaturization of Coupled Core Fluxgate Sensors
}

\author{
Jingjie $\mathrm{Li}^{1,2}$, Hadi Heidari ${ }^{3}$, Jiaqing Shi ${ }^{1,2}$, Yanzhang Wang ${ }^{1,2 *}$. \\ ${ }^{1}$ Key Laboratory of Geophysical Exploration Equipment, Ministry of Education, Jilin University, Changchun, China \\ ${ }^{2}$ College of Instrumentation and Electrical Engineering, Jilin University, Changchun, China \\ ${ }^{3}$ School of Engineering, University of Glasgow, Glasgow, the United Kingdom \\ yanzhang@jlu.edu.cn
}

\begin{abstract}
This paper demonstrates the probe structure optimization of coupled core fluxgate magnetic sensors through finite element analysis. The obtained modelling results have been used to optimize the probe structures from horizontal- to vertical- arrangements for magnetic crosstalk suppression and probe miniaturization. The finite element analysis show that with the same distance between each adjacent fluxgate elements, the magnetic crosstalk is suppressed by 6 times and the volume is reduced by 2 times after the optimization. Furthermore, the miniaturized probes with low magnetic crosstalk have been designed and implemented. The experimental results which showed more than 5 times suppression of magnetic crosstalk verified the simulation results. Therefore, the results provide detailed reference to cope with the contradiction between volume miniaturization and magnetic crosstalk suppression in magnetic sensor-array design.
\end{abstract}

Keywords-fluxgate magnetic sensor; resolution enhancement; crosstalk suppression; finite element analysis

\section{INTRODUCTION}

The magnetic sensors have emerged as a promising new sensing technology in various biosensing applications for detection, identification, localization and manipulation of a wide spectrum of biological, physical and chemical agents in the past few years [1-4]. More stringent requirements for sensors have been put forward due to the unique working environment of biosensing, such as higher spatial resolution, more compact structure, and lower power consumption under the premise of fine sensitivity and detection resolution [3-6].

The coupled core fluxgate magnetic (CCFM) sensor, based on the coupling-induced oscillations in overdamped bistable dynamics, has the great potential to achieve sub-pico Tesla detection. However, the bulky probe containing at least 3 elements constrains the sensor spatial resolution. And the magnetic crosstalk among adjacent elements inducing spurious signal makes CCFM far worse than its detection limit [7-12].

In this paper, the bulky horizontal arrangement was replaced by a slim vertical arrangement in the CCFM probe. The magnetic crosstalk were estimated by the finite element analysis (FEA) in ANSYS Maxwell. The results confirmed volume miniaturization and magnetic crosstalk suppression after the new arrangement utilized. The relationship between sensor volume and magnetic crosstalk of both VA and HA

This work was supported in part by the National Key Research and Development Plan (Code No. 2018YFC1503903), and Graduate Innovation Fund of Jilin University. ( ${ }^{*}$ Corresponding author: Yanzhang Wang) configurations has been analyzed to achieve a miniaturized probe with low magnetic crosstalk. In addition, the method to cope with the contradiction between volume miniaturization and crosstalk suppression is given and wish it can be a reference in magnetic sensor array design. Finally, the miniaturized probe with low magnetic crosstalk has been implemented for the future CCFM design.

\section{DESIGN AND METHODOLOGY}

\section{A. Vertical Arrangement for CCFM Probe}

The CCFM is constructed by unidirectional ring coupling $\mathrm{N}$ (in our design $\mathrm{N}=3$ ) fluxgate elements with cyclic boundary condition. Present CCFM probe is horizontal arrangement (HA) as shown in Fig. 1(a), and the fluxgate elements are placed on the surface of triangular support [7-9]. The huge volume waste mainly caused by the triangular support puts forward higher requirements for the spatial uniformity of the target magnetic field [13]. As shown in Fig. 1(b), the vertical arrangement (VA) for CCFM probe is proposed, and the fluxgate elements are installed along the central axis of cylindrical support.

\section{B. Details of CCFM Probe}

In Fig. 1, $r, L$ represent the radius and length of fluxgate element, and $d$ is the distance between adjacent fluxgate elements. Constrained by the process technology of the magnetic materials and to ensure the mechanical strength, the limit of dimension parameters are given as

$$
\left\{\begin{array}{c}
r \geq 2 \mathrm{~mm} \\
L \geq 20 \mathrm{~mm}
\end{array}\right.
$$

The magnetic core of CCFM is made of annealed Co-based amorphous numbered 2714A (Co-Fe-Ni-Si-B), the detailed features are listed in Table. I.

TABLE I. Details of Magnetic Core

\begin{tabular}{|c|c|c|}
\hline \multicolumn{3}{|c|}{ Dimension Parameters } \\
\hline Thickness & Width & Length \\
\hline $20 \mu \mathrm{m}$ & $1.0 \mathrm{~mm}$ & $20 \mathrm{~mm}$ \\
\hline \multicolumn{3}{|c|}{ Electrical Parameters } \\
\hline Magnetic Coercivity & Saturation Induction & Max. Permeability \\
\hline$\sim 2 \mathrm{~A} / \mathrm{m}$ & $\sim 0.57 \mathrm{~T}$ & $1,000,000 \mu_{0}$ \\
\hline
\end{tabular}



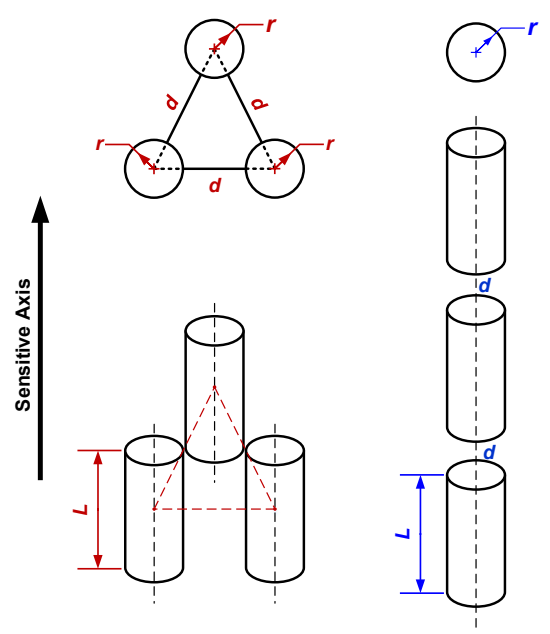

$\begin{array}{ll}\text { (a) Horizontal Arrangement } & \text { (b) Vertical Arrangement }\end{array}$

Fig. 1 Horizontal and vertical arrangement in CCFM probe.

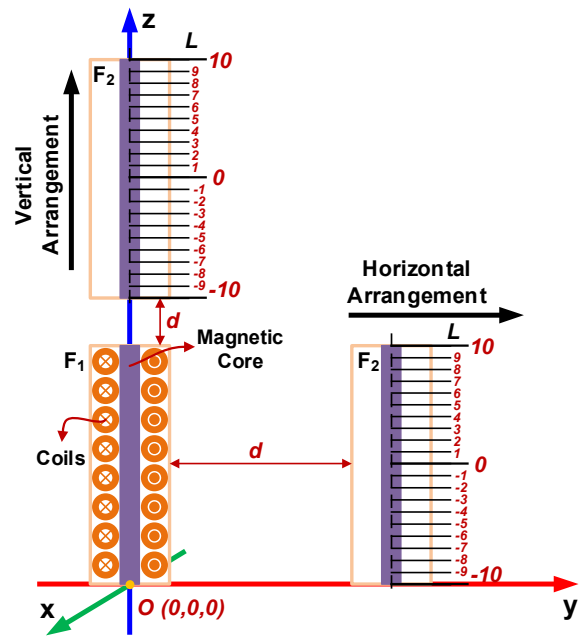

Fig. 2 The set-up of magnetic crosstalk evaluation.

\section{Magnetic CROSSTALK SuPPRESSION}

The CCFM is a complex dynamic system mainly due to the complicated hysteresis of the magnetic core. The characteristics of the coupling-induced oscillations in CCFM are very sensitive to parameters' perturbations, making CCFM a magnetic sensor with high sensitivity. However, the CCFM treats the magnetic crosstalk among fluxgate elements equally without any discrimination and results in partial saturation in the magnetic core which will generate a spurious signal and undesirable noise in the induction coil. Worse still, high magnetic crosstalk can break the oscillation conditions and prevent the CCFM from self-excitation, and then the sensor will stop working.

In this part, the magnetic crosstalk has been evaluated through the FEA in ANSYS Maxwell. Meanwhile, the sensor volume has been calculated and discussed.

\section{A. FEA Method for Magnetic Crosstalk Estimation}

The magnetic crosstalk is evaluated with the assistance of ANSYS Maxwell (FEA tools). And the parameters set in the
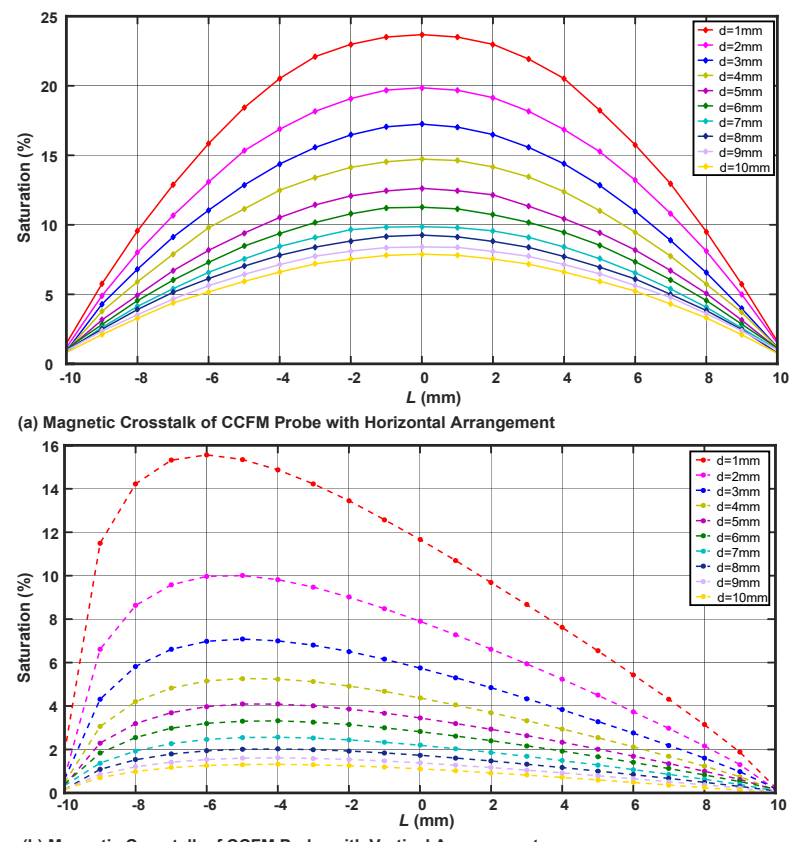

(b) Magnetic Crosstalk of CCFM Probe with Vertical Arrangemen

Fig. 3 Results of magnetic crosstalk obtained by ANSYS, and the saturation is defined as the ratio of crosstalk to saturation induction.

model are transferred according to Eq. (1) and Table. I. The detailed testing set-up is shown in Fig. 2 and described below:

1. The fluxgate elements are simplified as a combination of magnetic core and coils;

2. Set $r=2 \mathrm{~mm}, L=20 \mathrm{~mm}$, and the axial direction of the fluxgate element is $\mathrm{Z}$ axis;

3. Place one fluxgate element $\mathrm{F}_{1}$ at a determined position $O(0$, $0,0)$; For HA, element $\mathrm{F}_{2}$ at $(r, 0,0)$; For VA, $\mathrm{F}_{2}$ at $(0,0, L)$;

4. Give excitation field $10 \mathrm{~A} / \mathrm{m}$ (equivalent to the practical operation) to $F_{1}$ and leave $F_{2}$ unbiased;

5. For HA, $\mathrm{F}_{2}$ is moved along $y$-axis to set different distance $d$, $(0, d, 0)$; For $\mathrm{VA}, \mathrm{F}_{2}$ is moved along $y$-axis to set different distance $d,(0,0, d)$; The moving interval is set to be $1 \mathrm{~mm}$, and $\mathrm{d}$ is between $1 \mathrm{~mm}$ and $10 \mathrm{~mm}$;

6. Run Maxwell calculation and obtain the magnetic induction of $\mathrm{F}_{2} ; \mathrm{F}_{2}$ is marked with 21 points along its central axis.

The results with different distances $d$ are given in Fig. 3, and the maximum crosstalk of each distance $d$ is calculated and shown in Fig. 4. The results reveal that the magnetic crosstalk can be suppressed by $10 \%$ by optimizing the probe structure from HA to VA configuration. And for $d=10 \mathrm{~mm}$, the crosstalk of VA is more uniformly distributed along $L$ direction. As the

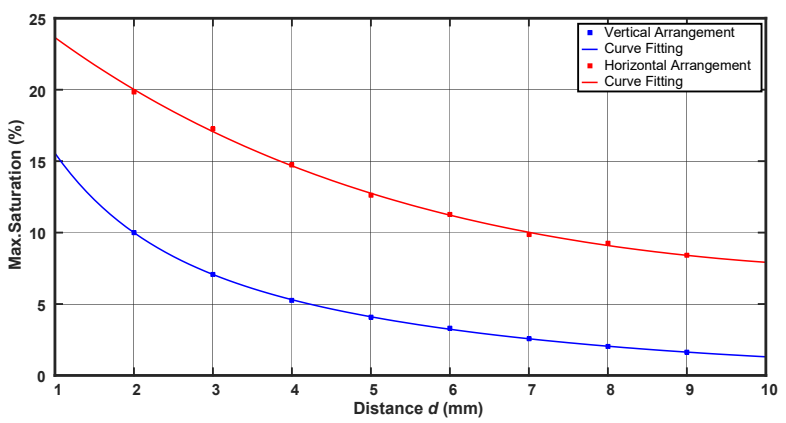

Fig. 4 Maximum magnetic crosstalk vs. distance $d$. 


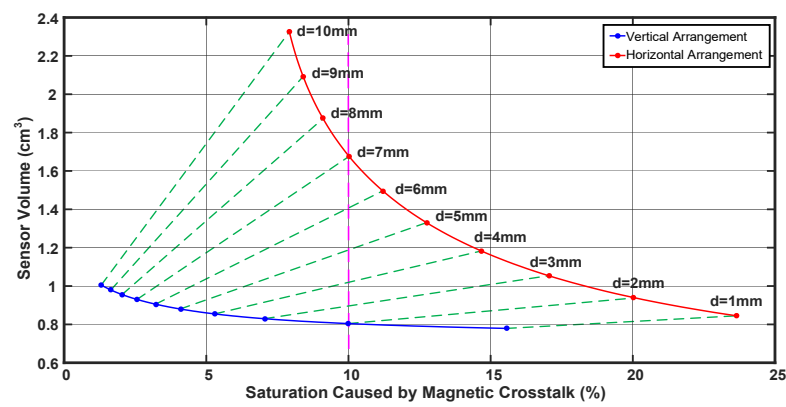

Fig. 5 Sensor volume vs. magnetic crosstalk.

distance $d$ increases, the crosstalk shows a tendency to become lower for both HA and VA configurations. Using $2^{\text {nd }}$ order exponential equation as target function for curve fitting, and getting the fitting equations,

$$
\left\{\begin{array}{l}
B_{H A}=26.41 \times e^{-0.1912 \times d}+1.683 \times e^{0.087 \times d} \\
B_{V A}=15.35 \times e^{-0.9717 \times d}+12.19 \times e^{-0.2234 \times d}
\end{array}\right.
$$

where $B_{H A}$ and $B_{V A}$ represent the magnetic crosstalk of HA probe and VA probe respectively.

\section{B. Sensor Volume Miniaturization}

The structure of HA probe is consisted of three cylinders and one triangular prism with half cylinder cross site. And the structure of VA probe can be viewed as a cylinder. Therefore, the sensor volume can be obtained by,

$$
\left\{\begin{array}{l}
V_{H A}=L\left[2.5 \pi r^{2}+\sqrt{3}(0.5 d+r)^{2}\right] \\
V_{V A}=\pi r^{2}(3 L+2 d)
\end{array}\right.
$$

where $V_{H A}$ and $V_{V A}$ represent the volume of HA probe and VA probe respectively.

Based on Eq. (2) and Eq. (3), the relationship between sensor volume and magnetic crosstalk is given in Fig. 5. It is shown that the VA probe structure has more potential to achieve low magnetic crosstalk with small volume than HA structure. When the distance $d$ is less than $10 \mathrm{~mm}$, the VA probe can obtain its smallest crosstalk $1.3 \%$ with a volume of $1.01 \mathrm{~cm}^{3}$, while the crosstalk of VA can only be reduced to $7.2 \%$ with a bulky volume of $2.33 \mathrm{~cm}^{3}$. In the process of reducing magnetic crosstalk by increasing the distance, the sensor volume increase of the probe with structure VA is smaller than that of probe with structure HA, which can be clearly verified by the two curve slopes in Fig. 5. This means the magnetic crosstalk can be furtherly reduced by the way of increasing the distance among each adjacent fluxgate elements, if necessary.

\section{PRoBe IMPLEMENTATION AND ESITIMATION}

It is clearly revealed that the sensor probe with VA structure has more advantages in terms of magnetic crosstalk and sensor volume. In order to obtain better magnetic crosstalk suppression with small volume, the distance among each adjacent fluxgate elements is set to be $10 \mathrm{~mm}$, and the CCFM probe with VA structure has been implemented and shown in Fig. 6. The magnetic core is placed inside the glass tube to prevent it from deformation. And the flxugate elements are installed on the central axis of the plastic support. In addition,

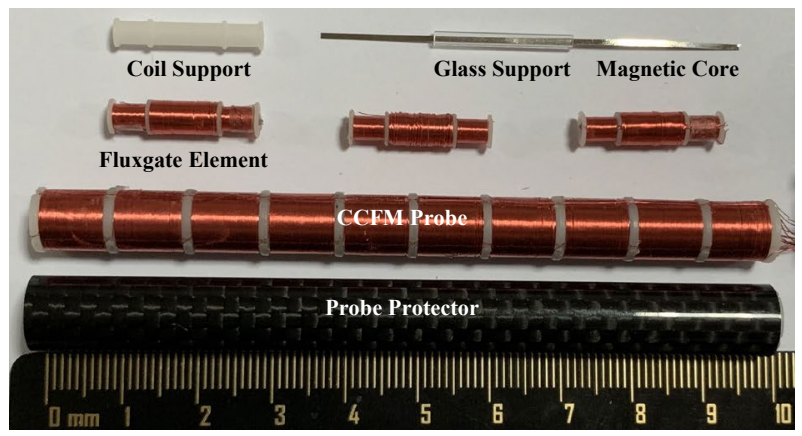

Fig. 6. Implemented CCFM probe with vertical arrangement structure.

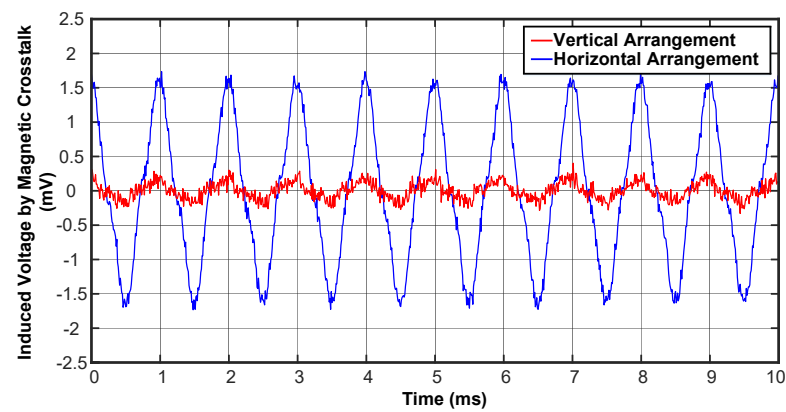

Fig. 7 Voltage induced by magnetic crosstalk of VA and HA probe $(\mathrm{d}=10 \mathrm{~mm})$.

the feedback and locking coils are wrapped arround the support to generate uniform magnetic fields along the central axis. Finally, the CCFM probe are protected by a carbon fiber tube.

The magnetic crosstalk has been evluated by the induced voltage excited by the adjacent fluxgate element. As shown in Fig. 7 , the induced voltage of VA probe $(0.65 \mathrm{mVpp})$ is about 5 times smaller than that of HA probe $(3.30 \mathrm{mVpp})$ which means the VA-structure probe has less magnetic crosstalk.

\section{CONCLUSION}

In this paper, the vertical arrangement structure for CCFM probe design is proposed. The FEA results revealed the benefits of magnetic crosstalk suppression and probe miniaturization brought by structure optimization. Finally, the miniaturized probe with low magnetic crosstalk has been implemented for CCFM design. Nowadays, we have found there are some detection defects in residence times differences fluxgate. And we have found the field compensation by feedback technology can solve this degradation [14]. This newly proposed CCFM probe with VA structure in this paper has potential to implement field compensation, because all the fluxgate elements are installed along the same axis.

\section{ACKNOWLEDGMENT}

The author deeply thanks Prof. Baglio Salvatore for the memorable discussion in IEEE Sensors 2018, New Delhi, India. His advise gave us inspirations to explore methods to enhance the spatial resolution (this manuscript) and to improve the detection strategy. 


\section{REFERENCES}

[1] V. Nabaei, R. Chandrawati, and H. Heidari, "Magnetic biosensors: modelling and simulation," Journal of Biosensors \& Bioelectron, vol. 103, pp. 69-86, December 2017.

[2] J. Ali, J. Najeeb, M. A. Ali, M. F. Aslam, and A. Raza, "Biosensors: their fundamentals, designs, types and most recent impactful applications: a review," Journal of Biosensors \& Bioelectron, vol. 8, pp. 1-9, January 2017.

[3] H. Heidari, "Electronic skins with a global attraction," Nature Electronics, vol. 1, pp. 578-579, November 2018.

[4] J. C. Rife, M. M. Miller, P. E. Sheehan, C. R. Tamanaha, M. Tondra, and L. J. Whitman, "Design and performance of GMR sensors for the detection of magnetic microbeads in biosensors," Sensors and Actuators A, vol. 107, pp. 209-218, November 2003.

[5] A. V. Reenen, A. M. D. Jong, J. M. J. D. Toonder, and M. W. J. Prins, "Integrated lab-on-chip biosensing systems based on magnetic particle actuation-a comprehensive review," Lab on a Chip, vol. 14, pp. 19661986, March 2014.

[6] A. Sandhu, "Biosensing: new probes offer much faster results," Nature nanotechnology, vol. 2, pp. 746-1-746-8, December 2007.

[7] P. Longhini, "Nonlinear dynamics design and operation of advanced magnetic sensors," The Claremont Graduate University, 2005.

[8] J. Li, Y. Wang, X. Zhang, C. Ji, J. Shi, "Sensitivity and Resolution Enhancement of Coupled-Core Fluxgate Magnetometer by Negative
Feedback," IEEE Transactions on Instrumentation and Measurement, vol. 68, no. 2, pp. 623-631, Feb. 2019.

[9] F. Antoci, B. Andò, C. Trigona, A. R. Bulsara, A. Nikitin, N.G. Stocks, and et al, "Injection locking in coupled core fluxgate magnetometers: Exploiting nonlinearity to enhance sensitivity to weak, low frequency, target magnetic fields," IEEE Sensors J., vol. 14, no. 2, pp. 554-562, Feb. 2014.

[10] M. Aguiar, P. Ashwin, A. Dias, and M. Field, "Dynamics of coupled cell networks: synchrony, heteroclinic cycles and inflation," Journal of Nonlinear Science, vol. 21, no. 2, pp. 271-323, Nov. 2011.

[11] A. Palacios, V. In, and P. Longhini, "Symmetry-breaking as a paradigm to design highly-sensitive sensor systems," Symmetry, vol. 7, no. 2, pp. 1122-1150, Jan. 2015.

[12] A. R. Bulsara, V. In, A. Kho, P. Longhini, A. Palacios, W. -J. Rappel, and et al, "Emergent oscillations in unidirectionally coupled overdamped bistable systems," Physical Review E, vol. 70, no. 3, Sep. 2004.

[13] M. Hernandez, V. In, P. Longhini, A. Palacios, A. Bulsara, A. Khob, "Coupling-induced oscillations in nonhomogeneous, overdamped, bistable systems," Physics Letters A, vol. 371, no. 24, pp. 4381-4387, Jun. 2008.

[14] J. Li, X. Zhang, J. Shi, H. Heidari, and Y. Wang, "Performance Degradation Effect Countermeasures in Residence Times Difference (RTD) Fluxgate Magnetic Sensors," IEEE Sensors J., unpublished, 2019. 